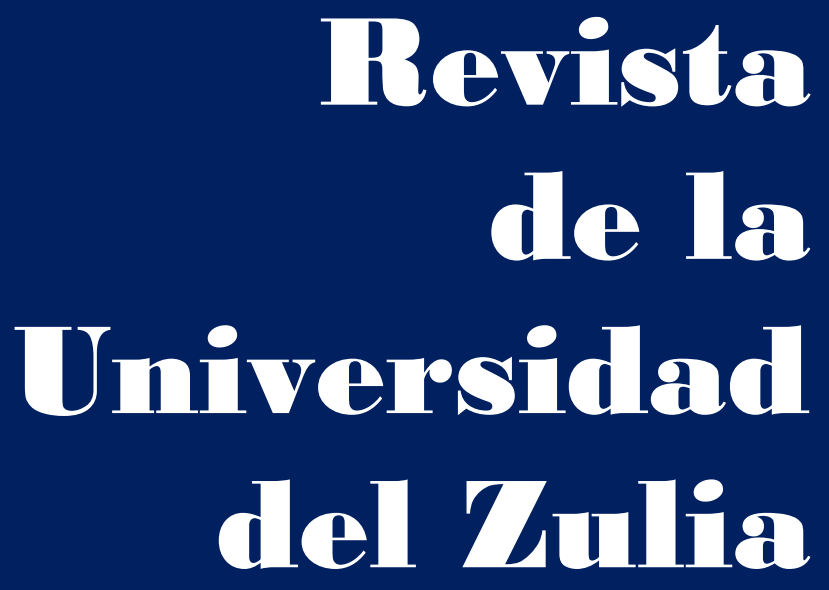

Fundada en 1947

por el Dr. Jesús Enrique Lossada

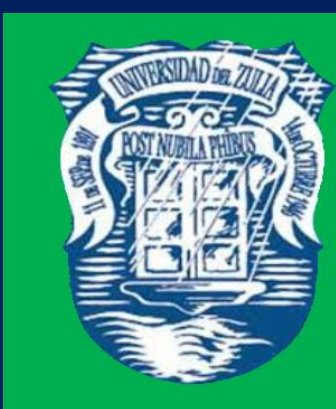

Ciencias del

Agred

Ingemieria

y Teenología
Añต 12 No 32

Enero - Abril 2021

Tercera Época

Maracailbo-Venezuela 
REVISTA DE LA UNIVERSIDAD DEL ZULIA. 3ㄹépoca. Año $12 \mathrm{~N}^{\circ}$ 32, 2021

Héctor Acacio Zerpa Ramírez et al. /// Modelo conceptual de productividad agrícola rural, 35-53

DOI: http://dx.doi.org/10.46925//rdluz.32.04

\title{
Modelo conceptual de productividad agrícola rural
}

\author{
Héctor Acacio Zerpa Ramírez * \\ Henry Izquierdo ** \\ Ricardo Chaparro ***
}

RESUMEN

Cientos de millones de familias rurales se ven atrapadas en un ciclo con baja productividad agrícola, dando lugar a un sufrimiento innecesario que obstaculiza el desarrollo agrícola y el crecimiento económico en general. La finalidad del artículo consiste en contribuir en la búsqueda de posibles soluciones para el problema de la baja productividad en el área agrícola, de las personas que habitan en los campos y en particular aquellos pobladores que se dedican y dependen de la agricultura como medio de subsistencia. El problema consiste en la pérdida de los derechos humanos, primordialmente en las poblaciones rurales. Teniendo en cuenta estas consideraciones, se planteó un marco conceptual y un enfoque analítico capaz de operacionalizar y por ende lograr establecer el modelo conceptual de productividad agrícola rural, con el objetivo de integrar y estudiar las variables que permitan explicar un efecto determinante sobre los ingresos de los productores, dichos ingresos son el resultado de aplicar un modelo que garantiza de forma natural, un equilibrio económico, social y ambiental. La investigación se enmarcó en el enfoque metodológico de tipo analítico. El modelo se planteó en función de estudios previos basados en modelos agroalimentarios, índices de progreso, modelos de productividad, análisis de las cadenas de valor, desarrollo endógeno, marcos conceptuales, enfoques analíticos y distritos industriales; permitiendo al modelo mencionado emerger como una metodología inédita denominado Modelo Conceptual de Productividad Agrícola Rural (MoPAR), el cual será una herramienta tecnológica que se entregará a los productores con la finalidad que sea incluido dentro de sus herramientas de toma de decisiones.

PALABRAS CLAVE: desarrollo endógeno, distrito industrial, cadena de valor, productividad, conglomerado, MoPAR.

*Profesor de la Universidad Nacional Experimental Politécnica Antonio José de Sucre UNEXPO. Estudiante del doctorado en Ciencias de la Ingeniería, UNEXPO, Venezuela. E-mail: hzerpa@unexpo.edu.ve

**Profesor titular jubilado de la Universidad Nacional Experimental de Guayana, Venezuela.

***Doctor en Investigación Agrícola. Instituto Nacional de Investigaciones Agropecuarias INIA, Venezuela.

Recibido: 02/06/2020

Aceptado: 11/09/2020 
REVISTA DE LA UNIVERSIDAD DEL ZULIA. 3época. Año 12 N³ 32, 2021

Héctor Acacio Zerpa Ramírez et al. /// Modelo conceptual de productividad agrícola rural, 35-53

DOI: http://dx.doi.org/10.46925//rdluz.32.04

\section{Conceptual model of rural agricultural productivity}

ABSTRACT

Hundreds of millions of rural families are caught in a cycle of low agricultural productivity, leading to unnecessary suffering that hinders agricultural development and economic growth in general. The purpose of the article is to contribute to the search for possible solutions to the problem of low productivity in the agricultural area, of the people who live in the fields and in particular those inhabitants who dedicate themselves and depend on agriculture as a means of subsistence. The problem is the loss of human rights, primarily in rural populations. Taking these considerations into account, a conceptual framework and an analytical approach capable of operationalizing and therefore achieving establish the conceptual model of rural agricultural productivity was proposed, with the objective of integrating and studying the variables that allow explaining a determining effect on the income of producers, said income is the result of applying a model that naturally guarantees an economic, social and environmental balance. The research was framed within the analytical methodological approach. The model was proposed based on previous studies based on agri-food models, progress indexes, productivity models, analysis of value chains, endogenous development, conceptual frameworks, analytical approaches and industrial districts; allowing the aforementioned model to emerge as an unprecedented methodology called the Conceptual Model of Rural Agricultural Productivity (MoPAR), which will be a technological tool that will be delivered to the producers in order to be included in their decision-making tools.

KEY WORDS: endogenous development, industrial district, value chain, productivity, conglomerate, MoPAR.

Introducción

Cientos de millones de familias rurales se ven atrapadas en un ciclo de hambre, pobreza y baja productividad que da lugar a un sufrimiento innecesario que obstaculiza el desarrollo agrícola y el crecimiento económico en general (FAO, 2015). Según el informe, Cómo evitar que la crisis del COVID-19 se transforme en una crisis alimentaria: Acciones urgentes contra el hambre en América Latina y el Caribe: Tras siete años de lento crecimiento, América Latina y el Caribe podría ver la mayor caída del PIB regional en un siglo (-5,3\%), lo que traerá en 2020 un aumento de la pobreza extrema de 16 millones de personas con respecto al año anterior, sumando 83,4 millones en total (CEPAL-FAO. 2020). La propuesta del artículo tiene como objetivo fundamental apoyar en la 
REVISTA DE LA UNIVERSIDAD DEL ZULIA. 3época. Año 12 N³ 32, 2021 Héctor Acacio Zerpa Ramírez et al. /// Modelo conceptual de productividad agrícola rural, 35-53

DOI: http://dx.doi.org/10.46925//rdluz.32.04

búsqueda de soluciones para el problema de la baja productividad, específicamente la población que habita en los campos y en particular aquellos pobladores que se dedican y dependen de la agricultura como medio de subsistencia. Los hogares rurales pobres obtienen sus ingresos de su propia producción o vendiendo su trabajo como mano de obra. Estos ingresos resultan inestables e insuficientes para la superación de la pobreza, por lo que se necesitan la aplicación de programas o planes que estabilicen y aumenten dichos ingresos. De manera que existe la explicita pérdida de derechos humanos, sustentado en lo establecido por Sen, como la privación de capacidades básicas y no meramente como la falta de ingreso (Sen, 2000).

Los marcos conceptuales (gestión social del riesgo, protección social transformativa, protección social adaptativa y la Iniciativa del piso de protección social) y los enfoques analíticos (enfoque de derechos, enfoque de ciclo de vida, enfoque operacional, enfoque de medios de subsistencia agrícola) tienen importantes aplicaciones en el desarrollo rural integral desde sus diferentes perspectivas, pero ninguno de ellos se centra específicamente en ellos como objetivo principal (FAO, 2019).

Se observa que existe un vacío en la manera como se abordan los marcos conceptuales y los enfoques, siendo estos aislados de la realidad, que se observa en las comunidades de agricultores, fundamentalmente, por la falta ausencia de la protección social plasmada en dichos marcos, así como la falta de instrumentos operaciones que realmente, permitan a las familias agricultoras, superar la pobreza. La investigación se enmarcó en el enfoque metodológico de tipo analítico.

El Modelo Conceptual de Productividad Agrícola Rural (MoPAR) emerge luego de analizar las cadenas de valor de Porter, cadenas agroalimentarias, desarrollo endógeno, los distritos industriales de Becattini, productividad, conglomerado, marcos conceptuales, que lograron realizar aportes muy específicos en cada aspecto analizado. Sin embargo, son aportes dispersos que no lograron revertir el ciclo de pobreza, abriendo una oportunidad de plantear una idea disruptiva en la que puedan converger, el marco conceptual mencionado, así como la propuesta de integración e interacción dedicadas a fortalecer la producción agrícola. 
REVISTA DE LA UNIVERSIDAD DEL ZULIA. 3a época. Año 12 N³2, 2021 Héctor Acacio Zerpa Ramírez et al. /// Modelo conceptual de productividad agrícola rural, 35-53

DOI: http://dx.doi.org/10.46925//rdluz.32.04

La estructura del presente artículo está formulada, en resumen, introducción, marco teórico, cuerpo principal, conclusiones, recomendaciones y referencias. La introducción menciona de manera muy resumida el problema, el alcance, los antecedentes, resultados y conclusiones. El marco teórico aborda los temas de interés tales como: distritos industriales, competitividad sistémica, productividad, cadenas de valor de Porter y agroindustriales. El desarrollo principal se aborda a través de la formulación del Modelo Conceptual de Productividad Agrícola Rural (MoPAR); por último, conclusiones, recomendaciones y referencias.

\section{Aspectos teóricos}

Para el desarrollo del modelo conceptual de productividad agrícola, que se presenta a continuación, se han estudiado diversas fuentes, habiendo quedado como las más relevantes desde la perspectiva epistemológica la relacionada con distritos industriales, representando esta, el elemento integrador entre el desarrollo endógeno y las potencialidades de una zona geográfica determinada, definido en los conglomerados. Igualmente, el hecho de contar con modelos de cadenas agroalimentarias predefinas, permite aplicar sobre dicha cadena el estudio de la cadena de valor de Porter, con la intención de encontrar las debilidades y fortalezas de los diferentes eslabones, permitiendo generar propuestas para la mejora de los procesos agrícolas. El objetivo del modelo de competitividad sistémica es transformar las ventajas comparativas en ventajas competitivas sustentables, lo cual queda representado en el hecho, que el 60\% de los alimentos consumidos en el mundo proviene de la pequeña agricultura de los países en desarrollo, quedando claro que muchos de estos pequeños productores, se manejan aplicando técnicas y semillas autóctonas y utilizando, abonos naturales, controlando plagas a través de líneas de cultivos anti plagas, poco uso de arados, entre otras; mostrando una alternativa que contribuye de manera directa con diversos objetivos del desarrollo sostenible.

\subsection{Distritos industriales}


REVISTA DE LA UNIVERSIDAD DEL ZULIA. 3ㄹépoca. Año $12 \mathrm{~N}^{\circ}$ 32, 2021 Héctor Acacio Zerpa Ramírez et al. /// Modelo conceptual de productividad agrícola rural, 35-53

DOI: http://dx.doi.org/10.46925//rdluz.32.04

Alfred Marshall, quien en su obra cumbre Principles of Economics, hace una observación determinante en la que explica que: las ventajas de la producción en gran escala pueden, en general, obtenerse bien agrupando en un mismo distrito un gran número de pequeños productores o bien construyendo pocas fábricas (Soler, 2008).

Esta afirmación viene a representar el origen de la teoría de los distritos industriales, sintetizando lo que será una clara línea de interés en el análisis económico: la unión entre la economía industrial y la percepción económica del territorio (Soler, 2008).

El distrito industrial, para Becattini, representa la entidad intermedia entre el sujeto económico singular y el sistema económico general, que asume la descripción de una comunidad local junto con la propia industria; esto es, una industria definida a través de la comunidad local (lo que ésta produce y el modo en el que organiza la producción), en lugar de a través de la tecnología productiva. Esto equivale a un vuelco del enfoque teórico tradicional de la investigación económica: en lugar de proceder desde la industria hacia su localización, se procede desde el lugar (donde propiamente se desarrolla la vida de las personas) hacia su industrialización (Sforzi, 2008).

Como puntualiza Michael J. Enright, uno de los colaboradores históricos de Porter, lo que distingue la noción de cluster de la de distrito es la presencia, en la definición del distrito, de la comunidad local, y complementando con el papel de factor de modificación de la productividad (Sforzi, 2008).

El distrito codificado por Becattini y por la escuela italiana es, ante todo, una comunidad local, el medio socio-cultural e institucional dentro del cual operan las empresas individuales y que constituye la condición de vida de las mismas (Sforzi, 2008).

Por otro lado, de la demanda, se extiende la superación de un estándar de confort habitual, la superación de dicho estándar crea las condiciones para el nacimiento de nuevos núcleos de necesidades de alto contenido social y cualitativo, que a su vez da lugar a demandas muy variables de productos diferenciados y personalizados (Becattini, 2004).

Un amplio y socialmente desigual crecimiento de la renta está concentrada en manos de un gran núcleo de clases medias que, alcanzando el estándar de confort del momento, se dedica 
REVISTA DE LA UNIVERSIDAD DEL ZULIA. 3a época. Año 12 N³2, 2021 Héctor Acacio Zerpa Ramírez et al. /// Modelo conceptual de productividad agrícola rural, 35-53

DOI: http://dx.doi.org/10.46925//rdluz.32.04

a la búsqueda de bienes y servicios cada vez más diferenciados y personalizados, aporten prestigio social. Estos fenómenos del mundo real señalan el nacimiento o el renacimiento de la nueva teoría de los distritos industriales (Becattini, 2004).

\subsection{Conglomerado}

Básicamente se trata de valorizar al máximo los recursos y las capacidades locales que se posean con objeto de identificar aquellas oportunidades que tengan en el marco de la Globalización, a partir de la especialización. Es cierto que cada país/región ha entendido el desarrollo local desde perspectivas diferentes (ordenación del territorio, empleo local, formación especializada, medio ambiente, innovación tecnológica, planes estratégicos, etc.); sin embargo, en aquellas regiones en las que se ha llegado a percibir ese desarrollo de una manera más intensa sobresale la figura del conglomerado de pequeñas empresas (pymes), especializadas en alguna actividad o proceso específico y que presentan ciertas posibilidades de desarrollo en un mercado abierto (Soler, 2008).

\subsection{Competitividad sistémica}

El modelo de competitividad sistémica plantea el desarrollo interfuncional e integral de seis niveles o subsistemas, con los que se sustenta el proceso competitivo de un país, región y empresa. El objetivo del modelo es transformar las ventajas comparativas en ventajas competitivas sustentables, elevando las capacidades competitivas de las empresas, los sectores productivos, las instituciones y el gobierno (Villarreal, 2001).

\subsection{Productividad}

La mayoría de los pequeños sistemas agrícolas son productivos, eficientes y sostenibles comparados con las grandes explotaciones, a pesar de su bajo uso de insumos químicos. La ventaja en rendimiento de los sistemas agrícolas diversificados puede variar entre 20 a 60 por ciento más alta que los monocultivos (Koohafkan \& Altieri, 2011).

Se lograron importantes aumentos de productividad a través de la "Revolución Verde" de las décadas posteriores a la Segunda Guerra Mundial, que se centraban en la cría de cultivos y 
REVISTA DE LA UNIVERSIDAD DEL ZULIA. 3ㄹépoca. Año $12 \mathrm{~N}^{\circ}$ 32, 2021 Héctor Acacio Zerpa Ramírez et al. /// Modelo conceptual de productividad agrícola rural, 35-53

DOI: $\underline{\text { http://dx.doi.org/10.46925//rdluz.32.04 }}$

ganado insensibles a insumos externos (por ejemplo, fertilizantes químicos y pesticidas, antibióticos, alimentos proteínicos) para prosperar en sistemas uniformes e intensivos. Si bien estos enfoques han producido aumentos significativos en la producción mundial de alimentos básicos, los rendimientos no mejoraron, se deterioraron o se derrumbaron en el 24-39\% de las zonas de producción de maíz, arroz, trigo y soja en las últimas décadas (Frison, 2016).

El uso masivo de plaguicidas asociado con la agricultura industrial ha llevado a problemas cada vez mayores de resistencia a las plagas, afectando los rendimientos y generando costos para los agricultores (por ejemplo, semillas y plaguicidas adicionales) (Frison, 2016).

Cómo se cultiva la tierra (no cuánto) puede de hecho ser la preocupación más urgente. Globalmente, el 20\% de la tierra se considera ahora degradada, y la agricultura industrial contribuye significativamente a esta tendencia (Frison, 2016).

Las comparaciones son cada vez más favorables a los sistemas diversificados cuando se comparan los resultados totales, en lugar de los rendimientos específicos de los cultivos. El 60\% de los alimentos consumidos en el mundo proviene de la pequeña agricultura de los países en desarrollo (Frison, 2016).

Tradicionalmente la modernización económica ha ido unida al desarrollo industrial, por lo que resulta difícil entender el proceso de cambio estructural almeriense basado en un sector «tradicional» como la agricultura (Ferraro \& Aznar, 2008).

\subsection{Cadenas de valor de Porter y cadenas agroalimentarias}

La cadena de valor, es un modelo teórico que describe la serie de tareas desarrolladas por una empresa para la elaboración de un producto o servicio que satisfaga las necesidades de sus clientes. La cadena de valor desagrega una empresa en sus actividades estratégicamente relevantes para entender el comportamiento de sus costes y las fuentes de diversificación existentes y potenciales. Una empresa obtiene ventaja competitiva mediante la realización de estas actividades estratégicamente importantes de forma más eficiente o mejor que sus competidores (Porter \& Stern, 2016).

Desde el punto de vista de la realidad socioeconómica, la cadena agroalimentaria es un sistema que agrupa actores económicos y sociales interrelacionados que participan 
REVISTA DE LA UNIVERSIDAD DEL ZULIA. 3ㄹépoca. Año $12 \mathrm{~N}^{\circ}$ 32, 2021

Héctor Acacio Zerpa Ramírez et al. /// Modelo conceptual de productividad agrícola rural, 35-53

DOI: http://dx.doi.org/10.46925//rdluz.32.04

articuladamente en actividades que agregan valor a un bien o servicio, desde su producción hasta que este llega a los consumidores (La Gra, 2016).

La cadena agroalimentaria puede ser interpretada desde el punto de vista analítico, como una forma de entender las relaciones entre actores en la agricultura y el medio rural, desde la provisión de insumos y la producción primaria hasta la entrega del producto al consumidor final, donde las relaciones que se establecen pueden ser de tipo contractual o comercial (La Gra, 2016).

\section{Metodología}

La investigación se enmarcó en el enfoque metodológico de tipo analítico. El modelo conceptual se planteó en función de estudios previos basados en modelos agroalimentarios, índices de progreso, modelos de productividad, análisis de las cadenas de valor, desarrollo endógeno, marcos conceptuales, enfoques analíticos y distritos industriales; permitiendo al modelo mencionado emerger, producto de análisis y diseño de ideas integradoras, como una representación inédita denominado Modelo Conceptual de Productividad Agrícola Rural (MoPAR), el cual será una herramienta tecnológica que será entregada a los productores con la finalidad que sea incluido dentro de sus herramientas de toma de decisiones.

\section{Modelo conceptual de productividad agrícola rural}

El Modelo Conceptual de Productividad Agrícola Rural (MoPAR) emerge luego de analizar las cadenas de valor de Porter, cadenas agroalimentarias, desarrollo endógeno, los distritos industriales de Becattini, productividad, conglomerado, marcos conceptuales, que lograron realizar aportes muy específicos en cada aspecto analizado. Sin embargo, son aportes dispersos que no lograron revertir el ciclo de pobreza, abriendo una oportunidad de plantear una idea disruptiva en la que puedan converger, el marco conceptual mencionado, así como la propuesta de integración e interacción dedicadas a fortalecer la producción agrícola.

Para modelar la actividad agrícola rural y estudiar la productividad, se propone un modelo inédito, cuyo objetivo fundamental es colocar al hombre como centro de estudio, y se configura de forma concéntrica en el modelo obtenido. Así, el modelo estudia el ser humano, a través del 
REVISTA DE LA UNIVERSIDAD DEL ZULIA. 3época. Año 12 N³ 32, 2021 Héctor Acacio Zerpa Ramírez et al. /// Modelo conceptual de productividad agrícola rural, 35-53

DOI: http://dx.doi.org/10.46925//rdluz.32.04

componente concéntrico mencionado y en adelante denominado Agro-Humano, como se muestra en la figura 1.

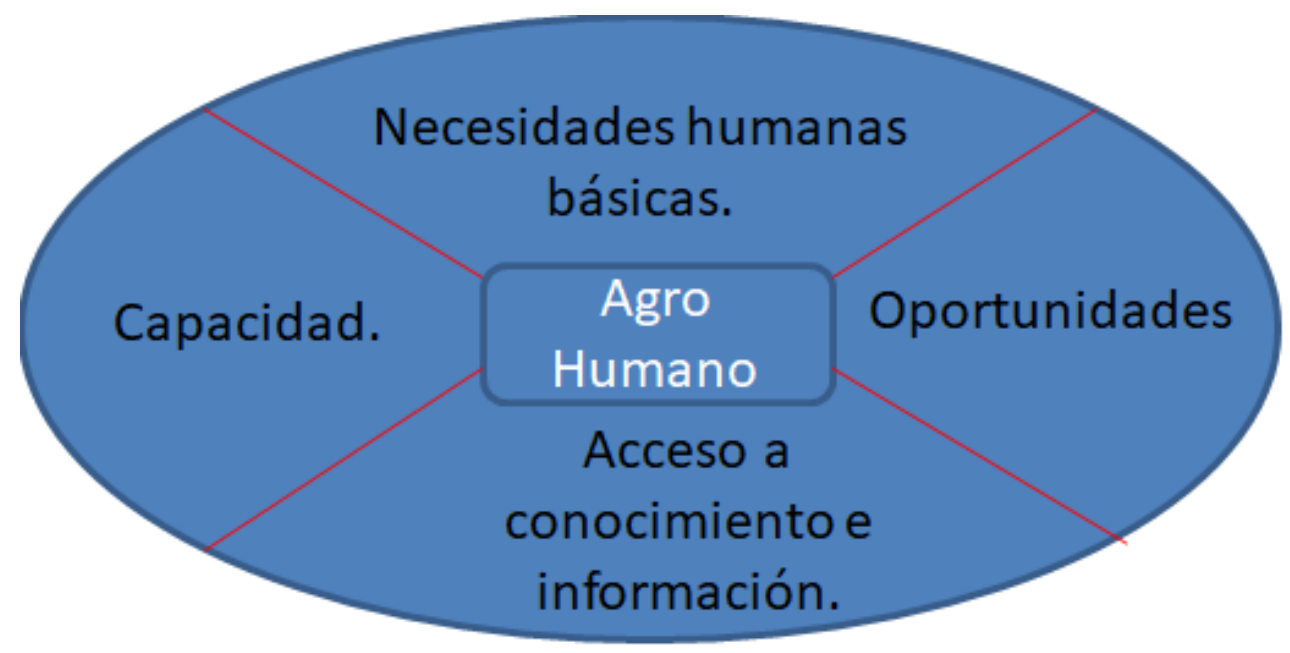

Figura 1. Componente concéntrico Agro-Humano.

Fuente: Elaboración propia.

El componente concéntrico Agro-Humano, tiene por finalidad, contener la información concerniente a indicadores que permiten mostrar valores referidos a necesidades humanas básicas, capacidad, oportunidades y accesos a conocimiento e información. Dichos datos tienen por finalidad validar los indicadores de gestión que muestran la influencia de los avances positivos o negativos de las decisiones tomadas en el modelo conceptual de productividad agrícola rural.

En la figura 2, se muestra la cadena agroalimentaria de productos agrícolas y se ve como un círculo que va desde la planificación de la producción, la cosecha y llega hasta la distribución a los consumidores, preparando de esta forma el terreno para el siguiente ciclo de planificación, producción, entre otros (La Gra, 2016).

La complejidad de la cadena agroalimentaria variará dependiendo de diversos aspectos, tales como el nivel de desarrollo del país, la localización geográfica, el tipo de cultivo, la época 
REVISTA DE LA UNIVERSIDAD DEL ZULIA. 3a época. Año 12 N³2, 2021 Héctor Acacio Zerpa Ramírez et al. /// Modelo conceptual de productividad agrícola rural, 35-53

DOI: http://dx.doi.org/10.46925//rdluz.32.04

del año, el clima, la tecnología disponible, la infraestructura, la oferta de mano de obra, y otros (La Gra, 2016).

Aunque la importancia relativa de los diferentes componentes de un sistema agroalimentario puede variar de acuerdo con el país y otros factores, una gran cantidad de ellos son comunes a la mayoría de las cadenas agroalimentarias (La Gra, 2016).

En la figura 2, se identifican 26 componentes. En algunos casos estos son de naturaleza institucional y se refieren a participantes tales como ministerios de agricultura, agricultores e intermediarios y los papeles que cada uno de estos desempeña en la cadena agroalimentaria. En otros casos, los componentes son de naturaleza funcional, como ocurre con la cosecha, el almacenamiento y el transporte, concentrándose en procesos o actividades que tienen lugar en un punto determinado del sistema (La Gra, 2016).

Cada uno de los referidos componentes poseen una cantidad variable de indicadores, los cuales han sido modificados, agregados nuevos, modificados otros y eliminados algunos de ellos, en presencia de expertos, que han sido consultados para efectos de garantizar la calidad y adaptabilidad del modelo conceptual de productividad a ser diseñado.

El Modelo Conceptual de Productividad Agrícola Rural, MoPAR (Ver figura 3), se diseñó a partir de la cadena de valor de Porter, en función del proceso de producción agrícola. Así, cada uno de los 26 componentes es potencialmente importante, debido a que las decisiones o acciones que se tomen en cada uno de los eslabones pueden afectar la producción, la productividad, la calidad o los costos del producto. Sin embargo, no todos los 26 componentes son relevantes para cada cadena agroalimentaria, en el sentido que, dependiendo del país de aplicación, de la época del año, del rubro a cosechar, entre otros, se puede obtener una cadena de valor, muy diferente, lo cual es natural en presencia de un modelo que puede ser utilizado para modelar eslabones que, aunque sean de rubros similares, cada eslabón puede ser diseñado de formas diferentes. Así, cumpliendo con las características de un modelo sistémico debe cumplir con propiedades tales como: equifinalidad, diferenciación y ser reproducibles. 
REVISTA DE LA UNIVERSIDAD DEL ZULIA. 3época. Año 12 N³ 32, 2021 Héctor Acacio Zerpa Ramírez et al. /// Modelo conceptual de productividad agrícola rural, 35-53

DOI: http://dx.doi.org/10.46925//rdluz.32.04

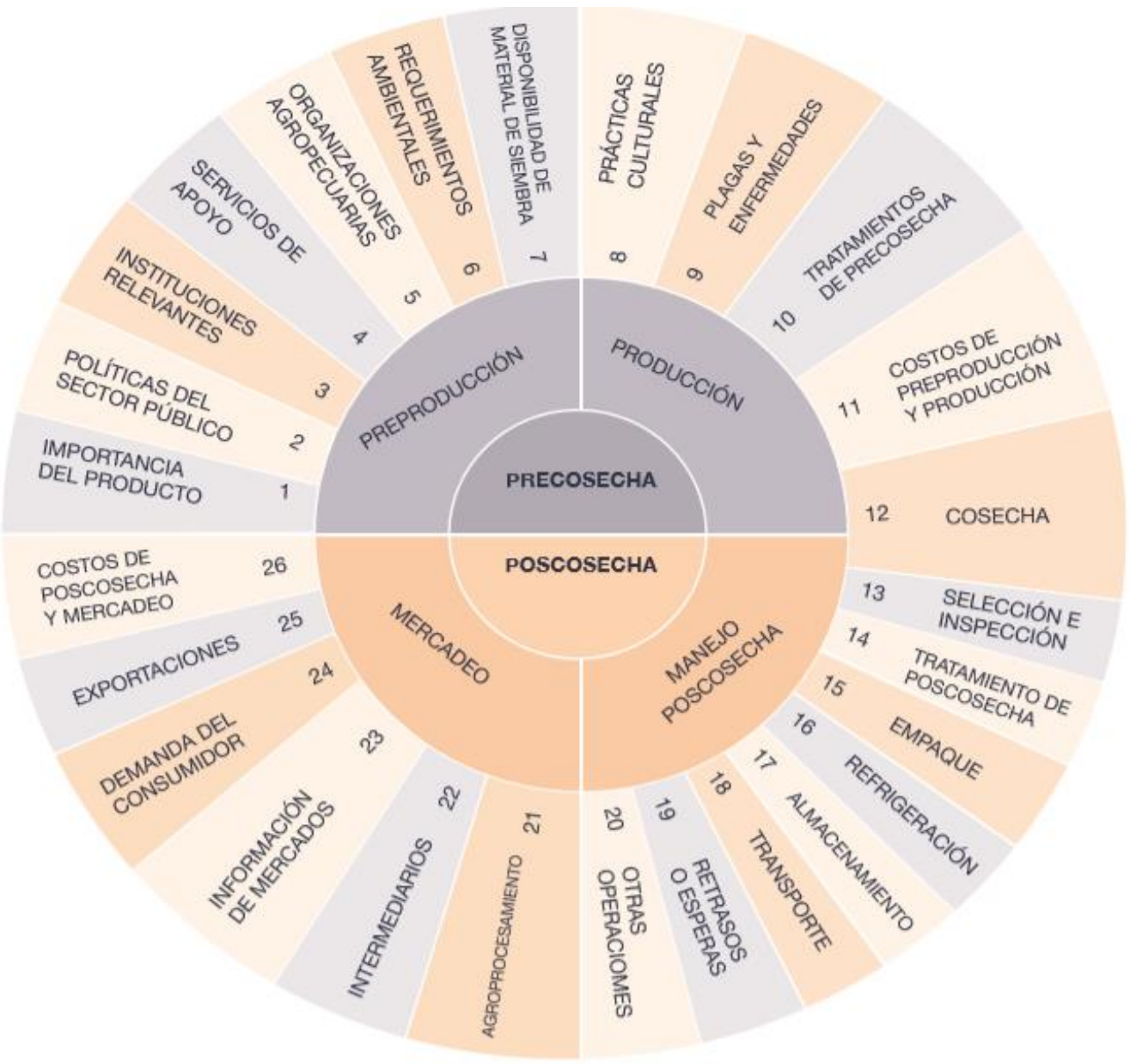

Figura 2. Componentes principales para la evaluación de cadenas agroalimentarias. Fuente: La Gra, J. (2016). IICA.

Asimismo, el modelo MoPAR, permite que los diferentes componentes puedan ser configurados, teniendo como fundamento, las bases teóricas que sustentan la investigación, tales como: desarrollo endógeno, conglomerado, distrito industrial, productividad, competitividad sistémica, marco conceptual, análisis de cadena de valor de Porter en los diferentes niveles de escala del estudio.

Así, el MoPAR se fundamenta en colocar como centro al ser humano y como dinamizador, el desarrollo endógeno y la sostenibilidad, que se practica en un lugar geográfico determinado, tomando en consideración que el sistema agrícola se basa en la agroecología. Por otra parte, el MoPAR, a través del análisis de la cadena de Porter, determina los eslabones de la cadena donde 
REVISTA DE LA UNIVERSIDAD DEL ZULIA. 3época. Año 12 N³ 32, 2021 Héctor Acacio Zerpa Ramírez et al. /// Modelo conceptual de productividad agrícola rural, 35-53

DOI: http://dx.doi.org/10.46925//rdluz.32.04

se requiera fortalecer la misma, entre ellas se puede mencionar agregar valor en uno o varios eslabones de la cadena, y el modelo plantea, cumplir con dicho requerimiento haciendo uso de innovaciones obtenidas de diferentes fuentes, entre las que podemos mencionar: centros de investigación de universidades, gubernamentales, privadas, experiencias propias, conocimiento trasmitido de generación en generación(conocimiento tácito), entre otras, de manera que se genera una transferencia de conocimiento, que permite determinar la mejor decisión, enmarcadas dentro de las metas de al menos nueve objetivos del desarrollo sostenible (ODS), entre ellos se encuentran, los mostrados en la Tabla 1, denominada Indicadores de Progreso Agrícola Rural (IPAR).

Los Indicadores de Progreso Agrícola Rural (IPAR), están constituidos por siete dimensiones y puede ser clasificado en cuatro categorías relativas a modelos de Venn: ambiental, social, económica y cultural. Las siete dimensiones están constituidas por un conjunto de componentes y/o indicadores según los cuales se pueden cuantificar los grupos en estudio, y por ende se puede validar el MoPAR, ajustados a los ODS. El IPAR se muestra en la tabla 1.

Es importante mencionar que la investigación ha sido planteada con la finalidad de apoyar a los agricultores o familias, que se encuentran en zonas rurales y que se encuentran en situación de pobreza.

Con este apoyo, se espera lograr un crecimiento interior, es decir, lograr el aumento de las capacidades de transformación, lo cual se puede evidenciar por los aumentos de producción, y por ende en mejorar la calidad de vida, así como lograr parte de los objetivos planteados en esta investigación, como lo es el desarrollo de un modelo conceptual de productividad agrícola rural, que sirva de apoyo, a la mejor toma de decisiones, por parte de los agricultores.

El crecimiento interior antes mencionado, sólo puede ser logrado bajo el enfoque del MoPAR, a través del desarrollo endógeno, las condiciones particulares de cada región geográficas, teniendo en cuenta el conocimiento propio del agricultor obtenido de generación en generación, por experiencias propias, y en muchos otros casos, gracias a los aportes de investigaciones provenientes de centros de investigación, tales como INIA, centros de investigación de las universidades, entre otras a nivel nacional e internacional. 
REVISTA DE LA UNIVERSIDAD DEL ZULIA. 3época. Año 12 N³ 32, 2021 Héctor Acacio Zerpa Ramírez et al. /// Modelo conceptual de productividad agrícola rural, 35-53

DOI: $\underline{\text { http://dx.doi.org/10.46925//rdluz.32.04 }}$

\begin{tabular}{|c|c|c|c|}
\hline $\begin{array}{l}\text { DIMENSIONES } \\
\text { DE IPAR }\end{array}$ & $\begin{array}{l}\text { DIMENSIONES DE } \\
\text { SOSTENIBILIDAD } \\
\text { (MoPAR) }\end{array}$ & INDICADORES & ODS \\
\hline Nivel de vida & Cultural/Social & $\begin{array}{l}\text { Agua segura para beber, electricidad, } \\
\text { combustible para cocinar, piso. }\end{array}$ & $1,2,10,11$ \\
\hline Salud ambiental & Ambiental & $\begin{array}{l}\text { Calidad agua, calidad suelo, calidad } \\
\text { aire. }\end{array}$ & $\begin{array}{l}3,6,13,14,1 \\
5,11\end{array}$ \\
\hline Salud Humana & Social & $\begin{array}{l}\text { Nutrición, mortalidad infantil, } \\
\text { Detección de enfermedades (TEST). } \\
\text { Muertes atribuibles a la } \\
\text { contaminación ambiental. } \\
\text { Oportunidades, capacidad, acceso a } \\
\text { conocimiento }\end{array}$ & $1,2,3,10,11$ \\
\hline $\begin{array}{l}\text { Costos de } \\
\text { Producción }\end{array}$ & $\begin{array}{c}\text { Ambiental/Social/Econ } \\
\text { ómica. }\end{array}$ & $\begin{array}{l}\text { Sueldos (empleos dignos hombre y } \\
\text { mujer), insumos (semilla, uso } \\
\text { agroquímicos) }\end{array}$ & $1,2,5,8$ \\
\hline Cosecha & Económico/Social & $\begin{array}{l}\text { Volumen de producto. } \\
\text { Porcentaje de producto apto para el } \\
\text { mercado. }\end{array}$ & 1,2 \\
\hline Comercialización & Económica & $\begin{array}{l}\text { Recursos transporte(carro, moto, } \\
\text { animal, humano). } \\
\text { Daños en el transporte. } \\
\text { Existen estadísticas de precio } \\
\text { disponibles. } \\
\text { Fuente de valor agregado. } \\
\text { Condiciones de pago. }\end{array}$ & 1,2 \\
\hline Mercados. & Económica. & $\begin{array}{l}\text { Manejo de precios. } \\
\text { control sobre los precios. } \\
\text { Consumo por grupos étnicos. } \\
\text { Variedad preferida, tamaño, sabor, } \\
\text { textura madurez, empaque, número } \\
\text { de unidades por paquete deseado y } \\
\text { color }\end{array}$ & $2,10,11$ \\
\hline
\end{tabular}

Tabla 1. Indicadores de Progreso Agrícola Rural (IPAR) y su convergencia con ODS.

Fuente: elaboración propia. 
REVISTA DE LA UNIVERSIDAD DEL ZULIA. 3a época. Año 12 N³2, 2021 Héctor Acacio Zerpa Ramírez et al. /// Modelo conceptual de productividad agrícola rural, 35-53

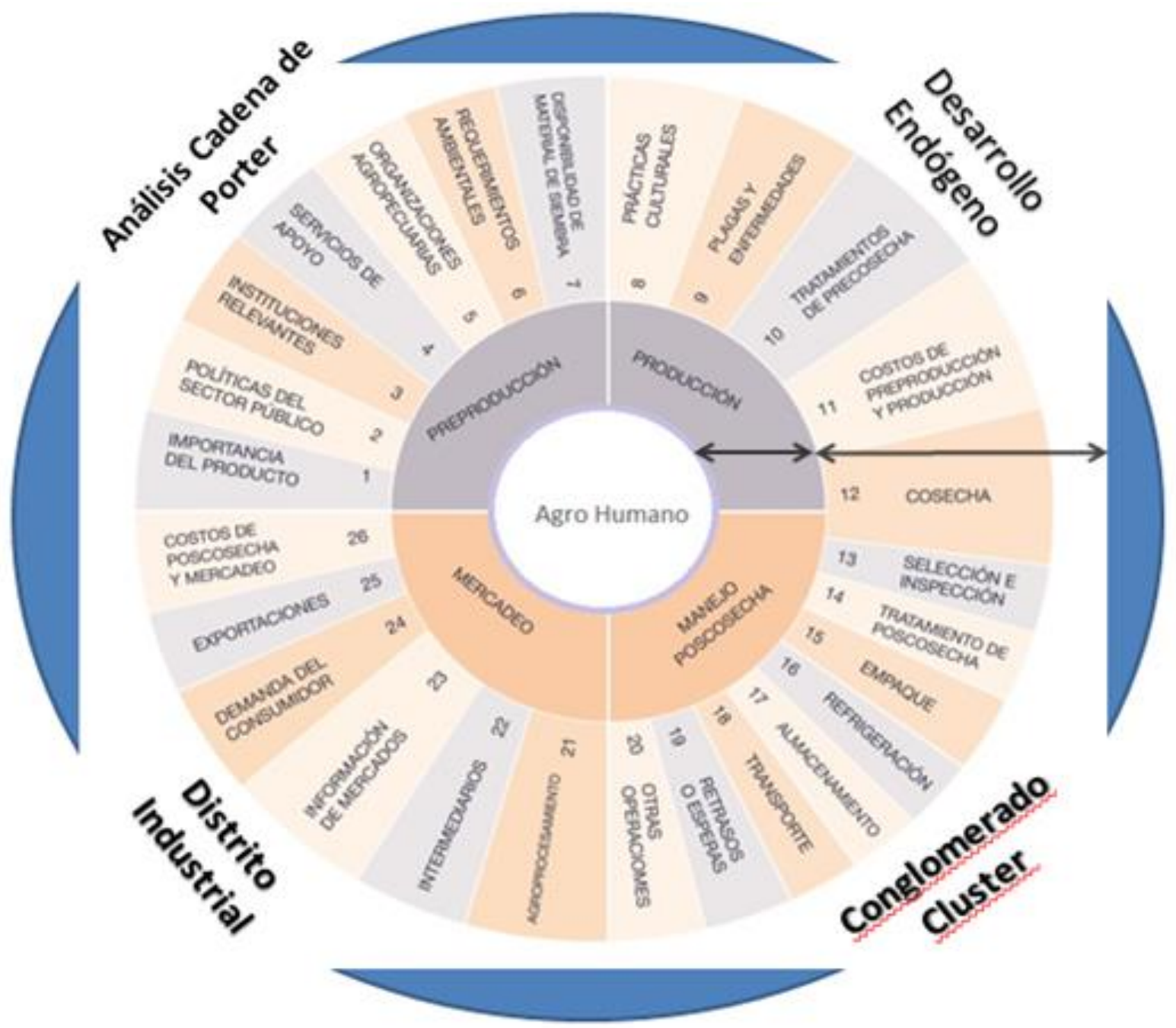

Figura 3. Modelo conceptual de productividad agrícola rural (MoPAR). Fuente: Elaboración propia.

La metodología para la implantación del MoPAR, por parte de los agricultores, debe aplicarse en diferentes fases a saber:

Fase 1: estudio y determinación de aspectos relativos a la precosecha. Aspectos fundamentales como la semilla, la cual debe cumplir con características agroecológicas que permitan hacer uso de semillas autóctonas que cuenten con un rendimiento igual o superior a las semillas comerciales y/o importadas. Es conveniente definir una forma de arado que permita 
REVISTA DE LA UNIVERSIDAD DEL ZULIA. 3época. Año 12 N³ 32, 2021 Héctor Acacio Zerpa Ramírez et al. /// Modelo conceptual de productividad agrícola rural, 35-53

DOI: http://dx.doi.org/10.46925//rdluz.32.04

cumplir con ciertas especificaciones de sostenibilidad. Estas consideraciones deben ser documentadas y sometidas a revisiones técnicas y cotejadas con el conocimiento tácito de los productores.

Fase 2: Transferencia de tecnología agrícola en ambos sentidos. Se pretende que pueda existir una realimentación entre productores, investigadores y demás actores, en la transferencia de tecnologías agrícolas aplicadas y que muestren los mejores rendimientos. Para ello, es posible comparar semillas propias de los productores y semillas de laboratorio proveniente de centros de investigación, con la finalidad de unificar criterios y esfuerzos, para mejorar los rendimientos y por ende aumentar la productividad.

Fase 3: Conocimiento del negocio. Para lograr comprender mejor el negocio agrícola es conveniente realizar a través de talleres, la transferencia de conocimiento, acerca de la cadena de Porter, con la finalidad de mostrar al productor como organizar su negocio desde la precosecha, cosecha y postcosecha. En cada fase se debe hacer un estudio económico con la finalidad de mantener una estructura de costos, que permita determinar los niveles de mejora o fracaso del modelo.

Fase 4: Transferencia de tecnología en Inteligencia de Negocios (BI). La propuesta contiene un software de inteligencia de negocios donde se puedan desarrollar indicadores, de diferente índole, con la finalidad de mostrar de manera gráfica, las tendencias y los valores obtenidos en las diferentes fases. Para entregar este conocimiento al productor se requiere de un plan de formación en campo.

Fase 5: Evaluación y refinamiento. Es una fase que permitirá a los actores a realizar evaluaciones en los diferentes eslabones de la cadena de valor con la finalidad de realizar mejoras, para refinar el modelo y por tanto, lograr el fin, mejora de la productividad agrícola rural, siguiendo los fundamentos de la sostenibilidad y contribuir con los ODS.

Así, a través de estas buenas prácticas, y con el uso del MoPAR, se espera que los productores, puedan realizar su toma de decisiones de una forma técnica, con la puesta en práctica de las potencialidades que brinda el modelo en estudio. El uso del modelo debe ser para el productor, tal que le brinde agilidad de visión del escenario que puede tener en un momento 
REVISTA DE LA UNIVERSIDAD DEL ZULIA. 3ㄹépoca. Año $12 \mathrm{~N}^{\circ}$ 32, 2021 Héctor Acacio Zerpa Ramírez et al. /// Modelo conceptual de productividad agrícola rural, 35-53

DOI: http://dx.doi.org/10.46925//rdluz.32.04

dado, sin hacer que cambie o modifique drásticamente, sus prácticas de cultivo, siempre y cuando las mismas se encuentren dentro de un marco conceptual, que cumpla con las expectativas, fundamentadas en el modelo generado.

Este tipo de prácticas, se espera que permita emerger a los productores como una fuerza de trabajo, es decir, como una organización o pequeña empresa, que pueda crecer como una unidad de producción y que a su vez, permita a los productores vecinos, utilizar las mismas experiencias, con la finalidad de entretejer y fortalecer las relaciones existente entre los productores, que permita configurar conglomerados, y a su vez entretejer entre los conglomerados, estructuras más complejas, que permitan, industrializar por regiones geográficas y lograr tal especialización y calidad en sus productos, que les capacite para competir, en primera instancia para satisfacer el consumo nacional y luego contribuir con productos de calidad certificada para exportación. A estas estructuras de industrias geográficas es lo que se conoce como distritos industriales.

Así, el fortalecimiento y el nacimiento de los conglomerados contribuyen con la formación de cluster, en los cuales, se manifiesta una mayor organización y por ende un fortalecimiento de los eslabones de la cadena, contribuyendo en la mejora de la productividad. Sin embargo, el cluster por ser una estructura de mayor complejidad y que requiere del apoyo de organizaciones y entes de nivel nacional, se deja como una recomendación para futuras investigaciones.

Es conveniente cerrar el escrito, con una generalización del modelo, el cual, tiene como objetivo, colocar al hombre como centro de la investigación, el cual debe ser considerado en la ecuación, y por ende, su calidad de vida, debe ser tomado en cuenta en cada una de la toma de decisiones en los eslabones que conforman el referido modelo, teniendo en consideración que cada una de las decisiones tomadas, afecta el sistema y por ende al hombre.

\section{Conclusiones}

Tomando en cuenta el objetivo planteado de diseñar un modelo conceptual de productividad agrícola rural y los resultados obtenidos se concluye lo siguiente: 
REVISTA DE LA UNIVERSIDAD DEL ZULIA. 3época. Año 12 N³ 32, 2021 Héctor Acacio Zerpa Ramírez et al. /// Modelo conceptual de productividad agrícola rural, 35-53

DOI: http://dx.doi.org/10.46925//rdluz.32.04

- Se logró diseñar el modelo conceptual de productividad agrícola rural (MoPAR) que consta de un nivel denominado AgroHumano, en el cual, se definieron componentes bien diferenciados que permite, realizar un seguimiento en la validación del modelo.

-El nivel de la cadena de valor del MoPAR, logra representar de manera clara y genérica los componentes, que constituyen la cadena de valor agroalimentaria, la cual, se adapta a consideraciones generales de diferentes organizaciones de producción, independiente del nivel de producción y de la escala de producción, es decir, es aplicable tanto a una unidad productiva, como a una organización de mayor escala.

-Se pudo configurar un modelo, en el cual, se obtiene un modelo emergente basado en el desarrollo endógeno, dejando intactas, las prácticas del agricultor, logrando de esta manera, hacer prevalecer que el desarrollo pueda surgir de manera natural, desde los cimientos de las prácticas propias del agricultor, hacia la conformación de redes entre productores, que permitan configurar modelos más complejos de producción, sin perder los productos que generan, basados en la agregación de valor en diferentes niveles de la cadena agroalimentaria, teniendo en cuentas la transferencia de conocimiento entre los actores.

-El modelo planteado puede ser utilizado bajo esquemas de producción agroecológica, pero también aplica a esquemas tradicionales (modelos de monocultivo).

-El uso del modelo debe permitir al productor agilidad de visión de escenarios, para una mejor toma de decisiones, sin hacer que cambie o modifique drásticamente, sus prácticas de cultivo, haciendo emerger a productores, que, utilizando las buenas prácticas, permita configurar conglomerados, siendo estas estructuras de industrias geográficas los disruptivos distritos industriales.

\section{Recomendaciones}

De los resultados y conclusiones obtenidos en este trabajo, se recomiendan las siguientes investigaciones como elementos desagregados en la elaboración de un producto más complejo, que debe ser entregado, como producto final, a los agricultores con la finalidad de mejorar la productividad agrícola. Dichas recomendaciones son las siguientes. 
REVISTA DE LA UNIVERSIDAD DEL ZULIA. 3a época. Año 12 N³2, 2021 Héctor Acacio Zerpa Ramírez et al. /// Modelo conceptual de productividad agrícola rural, 35-53

DOI: http://dx.doi.org/10.46925//rdluz.32.04

-Desarrollar herramientas de inteligencia de negocios para obtener un mayor soporte, en la toma de decisiones.

-Se recomienda incluir dentro de los modelos, aspectos dedicados a estudiar factores tales como: la resiliencia, el manejo de riesgos, entre otros; los cuales, contribuyen a fortalecer el MoPAR.

- Diseñar un índice de pobreza rural, que permita revelar de manera clara, las necesidades, que realmente padecen los centros de producción agrícolas, a nivel familiar.

-Para investigaciones de postgrados, se recomienda el estudio de la aplicación de inteligencia artificial, para la automatización de procesos, basado en la toma de decisiones, donde se consideren, aspectos que puedan dar respuestas anticipadas a eventos naturales tales como hambrunas, desastres naturales, fenómenos del Niño, fenómenos de la Niña, cambios de climas como nevadas, heladas, entre otros, que perjudican y generan pérdidas económicas tanto en parcelas sembradas como las consecuencias de falta de alimentos.

\section{Referencias}

Becattini, G. (2004). Del distrito industrial marshalliano a las "teoría del distrito" contemporánea. Una breve reconstrucción crítica. Investigaciones Rregionales. 1, 9-32.

CEPAL-FAO. (2020). Cómo evitar que la crisis del COVID-19 se transforme en una crisis alimentaria Acciones urgentes contra el hambre en América Latina y el Caribe.

FAO. (2015). El estado mundial de la agricultura y la alimentación. La protección social y la agricultura: romper el ciclo de la pobreza rural. Roma.

FAO. (2019). Curso: Pobreza Rural, Seguridad Alimentaria y Nutricional y Sistemas Inclusivos de Protección Social en América Latina y el Caribe.

Ferraro F. \& Aznar Á. (2008). El distrito agroindustrial de Almería: un caso atípico. Colección Mediterráneo Económico. Cajamar.

Frison E. (2016). IPES-Food report From Uniformity to Diversity: A Paradigm Shift from Industrial Agriculture to Diversified Agroecological Systems. [De la uniformidad a la diversidad]. www.ipes-food.org. 
REVISTA DE LA UNIVERSIDAD DEL ZULIA. 3época. Año 12 N³ 32, 2021 Héctor Acacio Zerpa Ramírez et al. /// Modelo conceptual de productividad agrícola rural, 35-53

DOI: http://dx.doi.org/10.46925//rdluz.32.04

Koohafkan, P. \& Altieri, M. (2011). Sistemas importantes del patrimonio agrícola mundial. Un legado para el futuro. FAO. Roma.

La Gra, J. (2016). Metodología de evaluación de cadenas agroalimentarias para la identificación de problemas y proyectos: un primer paso para la disminución de pérdidas de alimentos. San José - Costa Rica. IICA.

Porter, M. \& Stern S. (2016). Índice de progreso social. Incae Business School.

Sen, A. (2000). Desarrollo y Libertad. Barcelona: Editorial Planeta.

Sforzi, F. (2008). Unas realidades ignoradas: de Marshall a Becattini. Mediterráneo económico. Cajamar. España.

Soler, V. (2008). Preámbulo: los distritos industriales, como una oportunidad competitiva. Mediterráneo económico. Cajamar.

Villarreal R. (2001). México Competitivo 2020, Un Modelo de Competitividad Sistémica para el Desarrollo. Editorial Océano. México. 